de L'hydrate de sodium à 16 lires le kilo, serait de 3,6 centimes en utilisant des butyromètres Gerber et de 3 centimes seulement en se servant des butyromètres Morsin ou Höyberg. Ce prix est nettement inférieur à celui de 14-17 centimes qui est admis ordinairement pour les méthodes Gerber, Magliano, Höyberg et Neusal, à celui de 18-20 centimes de la méthode Sing, à celui de 20-30 centimes des méthodes Morsin et Cavalli.

\title{
ESSAIS SUR LA FABRICATION DU PORT-SALUT EN PARTANT DE LAITS PASTEURISÉS REEENEMENCÉS DE LEVAIN LACTIQUE
}

\author{
par CH. PORCHER † et G. THIEULIN
}

Les laits que l'on désire pasteuriser pour la fabrication du Port-Salut donnent parfois des fromages présentant d'assez nombreux trous, ce qui traduit la présence d'une quantité insolite de $B$. lactis aerogenes. Sans doute, le développement de ces derniers est-il facilité par une acidité moindre du lait, tenant vraisemblablement à des laits de mammites. Sur des laits d'acidité normale dont l'acidification part franchement, les ferments lactiques ordinaires font la police du milieu et, dans une certaine mesure, s'opposent au développement exagéré du $B$. lactis aerogenes. Mais, sur des laits malades, alcalins, dont l'acidification tarde, on est conduit à admettre que le $B$. lactis aerogenes, toujours présent dans ces laits, en même temps que le $B$. coli, trouve des conditions plus favorables pour son développement.

Il faut done d'abord s'assurer de la qualité des laits au point de vue de leur réaction et le mieux qu'on puisse faire ici pour ces laits destinés à la fabrication des fromages, e'est d'utiliser le réactif à l'alizarol. Rien n'empêche également de recourir à des catalasimètres plus ou moins rapides, mais, alors que l'épreuve à l'alizarol doit être réalisée sur les laits de tous les fournisseurs, l'épreuve catalasimétrique peut être plus espacée.

La quantité de présure utilisée est à peu près trois fois celle qui devrait être nécessaire pour un emprésurage normal, s'effectuant sur des laits de réaction également normale. Sans doute, en augmentant l'action de la présure, pense-t-on faciliter le caillage ; mais, à la réflexion, c'est l'acidité du lait au départ qu'il faut penser à améliorer. Sur des laits normaux, cette acidité, d'habitude, se déclanche bien et il suffit, pour la fabrication du Port-Salut, que l'acidité acquise ait atteint $2^{\circ}$ ou $3^{\circ}$, e'est-à-dire que l'acidité totale, en degrés Dornic, du lait, soit de 20 à $21^{\circ}$, pour avoir un emprésurage normal et une acidification qui parte bien. Mais, avec des laits alcalins, 
malades, qui infectent les cuves et, en quelque sorte, empoisonnent la masse, la réaction est au-dessous de la normale, pour des raisons pathologiques. Il faut done l'amener à une réaction normale et même au-delà, pour faciliter l'emprésurage.

Bien des moyens s'offrent à nous.

Nous avons pu penser à recourir à une acidité apportée dans le lait, non pas par un acide fixe comme l'acide lactique ou l'acide chlorhydrique, mais préférablement par un acide volatil comme l'acide carbonique, et les premiers résultats obtenus jusqu'ici sont assez favorables.

Si à cette acidité on ajoute un peu de chlorure de calcium, - mais il en faut peu -, on obtiendra la fermeté dans le caillé ou, selon la quantité de chlorure de calcium que l'on prendra, un caillé ayant une bonne consistance.

Il convient de rappeler que l'acidité est là pour favoriser l'action essentielle de la présure fonctionnant comme agent protéolytique et que le chlorure de calcium, lui, ne favorise que le second temps de l'emprésurage considéré comme processus complet, e'est-à-dire la coagulation. On peut ajouter dans les laits qui tardent à cailler, dans les laits paresseux parce qu'ils sont malades ou alcalins, du chlorure de calcium ; on ne facilitera pas l'emprésurage, comme on pourrait le penser, alors même que les doses de chlorure de calcium ajouté seraient plutôt élevées.

C'est donc toujours à l'acidité au départ qu'il faut penser, d'autant plus que les laits alcalins tendent à augmenter, parce que la mammite est envahissante, sous les formes plutôt chroniques et sub-chroniques qui, bien que peu cliniques, n'en altèrent pas moins la sécrétion et n'en modifient pas moins sa réaction biochimique vis-à-vis de la présure.

Un bon emprésurage est à la base de toutes les bonnes fabrications de fromages; c'est donc lui qu'il faut déterminer, dans les meilleures conditions possibles.

On pourrait penser que l'addition d'un bon levain lactique dans du lait même non pasteurisé, faciliterait, d'une part l'emprésurage, d'autre part la maturation du fromage. La chose a d'ailleurs été essayée, répétons-le, sans que le lait fût pasteurisé. Mais, pour qu'elle réussise, il faut que l'on apporte dans le lait une assez grande quantité de levain lactique, pour deux raisons :

a) d'abord, pour apporter de l'acide lactique dans le lait, - indépendamment, par conséquent, de toute considération microbienne pour en augmenter l'acidité, ce qui, par cela même, favorise l'acte essentiel de l'emprésurage, le premier temps, l'action sur la caséine ; b) ensuite, parce qu'on inonde la cuve d'une culture de bons ferments lactiques, - en admettant, bien entendu, que le levain soit excel- 
lent, - on apporte done d'excellents germes qui joueront le rôle de bons ferments en même temps qu'ils contrebattront l'influence des mauvais qui, dans le fromage Port-Salut, sont plutôt du groupe Coli-aerogenes.

Cette technique, malgré tout, n'a pas toujours eu de bons résultats parce que, avec des laits très infectés, très sales, d'une réaction plutôt alcaline, l'apport d'un bon levain lactique ne se faisait pas en proportion suffisante pour contrebalancer les facteurs nocifs que nous venons d'évoquer.

C'est pourquoi, on a pensé à pasteuriser le lait.

La pasteurisation du lait se présente à nous sous un angle favorable, mais, si elle a ses avantages, elle a peut-être aussi ses inconvénients.

L'avantage, c'est de détruire une flore indésirable. Cette flore indésirable est d'une assez grande résistance pour les fromages à pâte cuite, parce qu'il s'agit souvent de ferments butyriques. Mais pour les fromages à pâte très légèrement cuite comme le Port-Salut, la flore indésirable est faite d'éléments comme le Coli et surtout le $B$. lactis aerogenes, beaucoup plus dangereux que le Coli, dont la résistance à la chaleur est relativement peu marquée.

La pasteurisation, en détruisant cette flore indésirable assez peu résistante, n'est pas sans toucher vigoureusement aussi aux bons ferments lactiques. La stassanisation semble bien présenter, de ce côté, un avantage : celui de ne pas détruire complètement la flore lactique, la bonne, de laisser certains échantillons de ferments lactiques vivants, en se contentant de les amoindrir dans leur vitalité qu'ils retrouveront complète avec le temps.

Il serait souhaitable que la pasteurisation fût faite à la température la plus basse possible. On pourra commencer par $75^{\circ}$, mais il faudra voir si nous ne pourrions pas descendre à 73 , puis 72 et peut-être même à $70^{\circ}$; par cette température, nous atteindrons sûrement le Coli et l'aerogenes, mais nous toucherons moins aux ferments lactiques originels. De cette façon, quand nous ajouterons du levain lactique à la cuve, son développement pourra entraîner celui des ferments lactiques amoindris, endormis, mais non tués.

Un des inconvénients de la pasteurisation en général, dans la fromagerie, e'est de porter atteinte à la flore lactique originelle, à laquelle, sans se l'expliquer d'une façon très claire, les fromagers tiennent tant.

Il est done indiqué, si l'on pasteurise, de réensemencer le lait, après l'action de la chaleur, avec des ferments lactiques de même qualité biochimique que ceux du lait originel.

Les levains lactiques seront préparés avec du lait écrémé, mais stérilisé, au moins 24 heures à l'avance, de façon que l'on ait un 
levain ayant au moins $100^{\circ} \mathrm{D}$. d'acidité, c'est-à-dire $80^{\circ}$ d'acidité acquise, correspondant à $8 \mathrm{gr}$. d'acide lactique par litre. Dans ces conditions, si l'on emploie 1 litre de ce levain pour 100 litres de lait pasteurisé, c'est ajouter à chaque litre du lait, presque $1^{0}$ Dornic.

Ceci est fort bien si le lait est de réaction normale; ce n'est pas suffisant si le lait est plutôt alcalin. C'est pourquoi il serait très bien que nous apportions au préalable une acidité minérale qui élève la réaction du lait de $1^{\circ}$ à $2^{\circ} \mathrm{D}$. et avec elle, un peu de chlorure de calcium, le lait étant alcalin, enfin, l'acidité du levain lactique. Nous aurons ajouté toutes ces acidités, mais en prenant bien garde de ne pas nuire à la consistance du caillé, que nous voulons toujours souple et non sec.

Il est une acidité qu'il nous faudra toujours apporter parce que nous lui joignons les microbes nécessaires, e'est l'acidité du levain. De ce côté donc, nous élevons l'acidité du lait régulièrement d'environ $1^{\circ} \mathrm{D}$. Mais, si ce n'est pas suffisant et qu'il nous faille ajouter encore une acidité supplémentaire, ce serait peut-être une erreur que de la chercher uniquement dans le levain lactique, ce qui nous obligerait à utiliser une plus grande quantité de celui-ci.

Nous verrions très bien qu'au lieu d'un litre on employât un litre et demi, mais pas plus.

Bref, derrière les grandes lignes que nous indiquons, nous voyons que nous avons, pour améliorer la fabrication du Port-Salut, plusieurs moyens à notre disposition :

10 Augmentation de l'acidité par des moyens chimiques, tels que l'apport d'acide carbonique ;

$2^{\circ}$ Facilité apportée à l'emprésurage par un peu de chlorure de calcium ;

30 Augmentation de l'acidité par des moyens biologiques, e'està-dire par le levain lactique ;

$4^{0}$ Apport de bons ferments sélectionnés, par les mêmes levains.

Les levains sont faits dans une cuve spéciale; le lait est légèrement agité pendant que les ferments s'y développent, si bien que lorsque la coagulation doit se produire, elle se fait simplement en petits grumeaux.

Il serait à conseiller que ces levains fussent jetés dans la cuve après avoir été tamisés, de façon à retenir les plus gros caillés qui n'auraient pas été amenuisés.

En nous basant sur ces considérations générales nous avons effectué des premiers essais et nous donnons maintenant le rapport de ces essais ainsi que les réflexions qu'ils nous ont inspirées.

Nous avons conseillé les opérations qui portaient sur quatre cuves : la cuve $n^{\circ}$ I contenant du lait qui a été travaillé comme 
d'habitude, sans que nous ayons fait intervenir ni l'acide carbonique ni le chlorure de calcium; température et quantité de présure habituelle.

Nous appellerons cette première cuve, la cuve $R$, parce qu'on n'a rien ajouté au lait.

La seconde cuve a été traitée par l'acide carboniqué; nous l'appellerons cuve $\mathrm{CO}^{2}$.

La troisième cuve a été additionnée de chlorure de calcium, puis on y a fait barboter de l'acide carbonique. Nous l'appellerons cuve $\mathrm{CaCl}^{2}-\mathrm{CO}^{2}$.

La quatrième cuve, enfin, n'a reçu que du chlorure de calcium. Nous l'appellerons done, ouve $\mathrm{CaCl}^{2}$.

Dans les tableaux qui suivent, nous rassemblons succinctement les renseignements que nous avons pu recueillir au cours du travail du lait. Indiquons, dès maintenant, le plan de travail qu'il faut suivre en prenant la surveillance des opérations:

1o Prendre l'acidité du lait de la cuve avant le commencement du travail, avec l'alizarol et avec la soude Dornic;

$2^{\circ}$ Prendre l'acidité du sérum de la même euve, après le travail, avec l'alizarol et avec la soude Dornic ;

$3^{\circ}$ Faire effectuer le dosage de la matière grasse d'un échantillon du lait entier de la cuve;

$4^{\circ}$ Effectuer la même opération sur le sérum ;

$5^{\circ}$ Dans le cas où l'on utilise l'acide carbonique, prendre l'acidité du lait, avant l'emprésurage bien entendu et après le passage du courant gazeux ;

$6^{\circ}$ Dans le cas où l'on emploie le chlorure de calcium, prendre l'acidité du lait de la cuve avant et après l'addition de ce sel, toujours avec l'alizarol et avec la soude Dornic ;

$7^{\circ}$ Dans les mêmes conditions, prendre l'acidité du sérum avec l'alizarol et la soude Dornic ;

$8^{\circ}$ Dans le cas où l'on utilise le chlorure de calcium, d'une part, et le gaz carbonique, d'autre part, on prendra l'acidité du lait avant et après l'addition de $\mathrm{CaCl}^{2}$, car e'est par là que l'on commencera, puis après le passage du gaz, et c'est par là que l'on terminera, juste avant d'ajouter la présure.

Bien entendu, on prendra également l'acidité du sérum, parce qu'ici on a utilisé le chlorure de calcium.

Nous ne pensons pas que l'acide carbonique puisse modifier la réaction du sérum, parce que, dans les conditions où l'on travaille le lait, l'acidité qu'il provoque, nécessaire à l'emprésurage, peut avoir disparu. En tout cas, si l'on y tient, il sera indiqué de prendre aussi l'acidité du sérum avec l'alizarol et avec la soude Dornic, alors qu'on n'a utilisé qu'un courant de $\mathrm{CO}^{2}$. 
$9^{\circ} \mathrm{Ne}$ pas oublier de doser également la matière grasse sur le sérum, dans les diverses conditions où l'on opère. Comme le chlorure de calcium et l'acide carbonique tendent à provoquer la formation d'un caillé plus ferme, on doit penser, a priori, que davantage do matière grasse est retenue dans le caillé et qu'il en reste par conséquent moins dans le sérum. Cette conclusion ne peut pas toujours être tirée des opérations d'une même journée, paree que le lait d'une cuve ne ressemble pas à celui des autres. On travaille le lait au fur et à mesure qu'il arrive.

La conclusion que nous venons de laisser espérer ne peut être tirée que d'un ensemble d'observations dans lesquelles on confrontera, d'une part le lait entier de la cuve avant le travail et le sérum de la même cuve après le travail du caillé, d'autre part.

Nous avons constaté que la quatrième cuve est toujours nettement plus acide que la première, l'acidité de chaque cuve allant en croissant, de la première à la quatrième.

Le travail de la première cuve a commencé aux environs de 9 heures et le travail de la quatrième, à midi. Trois heures se sont écoulées, et c'est un temps suffisant, avec un lait en pleine culture lactique, pour que son acidité se développe considérablement.

\section{Première cuve (R).}

Emprésurage à $38^{\circ}$.

On a chauffé jusqu'à $43^{\circ}$ pendant le brassage.

L'emprésurage a commencé au bout de 15 minutes.

La quantité de présure (présure Hansen) a été de $300 \mathrm{~cm}^{3}$ pour 850 litres.

Le caillé obtenu est plutôt mou.

Le rompage s'est fait en grains assez petits.

Quand on prend le caillé entre les mains et qu'on le serre, l'eau s'en va difficilement et l'on sépare le caillé pressé, un peu sec, non pas en grains, mais en petites masses.

A la sortie des presses, les fromages de cette cuve étaient durs, par comparaison avec ceux des autres cuves qui étaient mous.

Ceci peut surprendre et nous montre que les cuves, au point de vue de la qualité des laits, sont très différentes.

\section{Cuve $\mathrm{n}^{\circ} 2\left(\mathrm{CO}^{2}\right)$}

On a emprésuré à une température plus basse : $35^{\circ}$.

On a pris la même quantité de présure que pour la cuve $n^{\circ} 1(R)$; en effet, comme il n'y avait que 800 litres, on a pris $280 \mathrm{~cm}^{3}$.

On a fait passer le courant d'acide carbonique pendant 10 minutes. 
L'acidité, avant le passage gazeux, était de $18^{\circ} 1 / 2 \mathrm{D}$.; après le passage, de $20^{\circ} \mathrm{D}$.

Le lait s'est pris en 11 minutes.

La température de brassage n'a pas dépassé $40^{\circ}$.

Incontestablement, le grain était beaucoup plus ferme.

Après l'avoir pressé dans la main pour en évacuer l'eau, on pouvait le résoudre en grains assez compacts, d'une façon relativement facile.

\section{Troisième cuve $\left(\mathrm{CO}^{2}+\mathrm{CaCl}^{2}\right)$.}

Acidité du lait au départ : $19^{\circ} 1 / 2 \mathrm{D}$.

Après l'addition de chlorure de caleium, à la dose de 0 gr. 25 par litre (il ne s'agit pas de $\mathrm{CaCl}^{2}$ fondu, mais de $\mathrm{CaCl}^{2}$ desséché, qui renferme encore une certaine quantité d'eau de constitution), l'acidité monte à $20^{\circ} \mathrm{D}$.

Après le passage de $\mathrm{CO}^{2}$ pendant 10 minutes, l'acidité monte à $21 \frac{1}{2} \mathrm{D}$.

L'emprésurage a été réalisé à $35^{\circ}$.

La prise du caillé a eu lieu en 7 minutes.

Le brassage s'est fait jusqu'à $38^{\circ}$ seulement.

On a eu un caillé ferme, que nous pouvions même craindre un peu trop ferme.

Les fromages obtenus avec les laits de cette cuve sont mous.

\section{Quatriè me cuve $\left(\mathrm{CaCl}^{2}\right)$.}

Lait dont l'acidité n'a pas été prise au début, ce qui est. regrettable ; mais on peut dire, a priori, que c'était un lait très acide.

L'emprésurage a eu lieu à $12 \mathrm{~h}$. 30. C'était done la dernière cuve.

Ce lait est le plus acide, comme cela a toujours été constaté.

Il nous souvient également que nous avons mis du lait qui, avec l'alizarol, donnait une teinte jaune serin ; c'était done un lait extrêmement acide.

Le lait de cette cuve n'a été additionné que de $\mathrm{CaCl}^{2}$, toujours à la même dose : $0 \mathrm{gr}, 25$ au litre.

Il y avait 1.000 litres dans la cuve.

L'emprésurage a été fait à $35^{\circ}$.

La prise a eu lieu en 5 minutes.

Le grain était sec.

Les fromages de cette cuve, semblables à ceux de la cuve $\mathbf{n}^{0} \mathbf{2}$, étaient moins durs que ceux de la cuve $n^{0} 1$ (R).

Voyons les observations que peut nous suggérer l'examen du travail du lait dans ces quatre cuves. 
Il est surprenant que le lait de la euve $3, \mathrm{CO}^{2}+\mathrm{CaCl}^{2}$, qui nous a đonné un grain bien ramassé, ferme, ait abouti à des fromages mous, souples, élastiques, plus que ceux des trois autres cuves, alors qu'on aurait pu prévoir le contraire. Cependant, à la réflexion, lorsque nous avons un bon emprésurage, d'une façon générale, le caillé est toujours très élastique.

Il faudra recommencer les opérations dans le même ordre, en notant tout, comme nous l'avons dit un peu plus haut.

Nous avons même oublié, dans notre énumération ci-dessus, tout ce qui concerne le travail proprement dit du lait de la cuve. Il faudra done, aux indications déjà données, ajouter celles-ci :

10 Suivre attentivement le moment où, à partir de la mise en présure, le caillé commence à se prendre. C'est ce point qui, à notre avis, a le plus d'importance. Il marque vraiment le moment du départ de l'emprésurage :

$2^{\circ}$ Noter le moment où l'on eommence à rompre, puis à brasser ;

$3^{\circ}$ Bien noter la température d'emprésurage ;

$4^{\circ}$ Bien noter la quantité de présure ;

$5^{\circ}$ Bien noter la température maxima à laquelle on chauffe pendant le brassage, et le temps du brassage.

Toutes ces indications s'uniront étroitement à celles qui ont été déjà formulées, et en dépendront, en quelque sorte.

Il est certain que des prises rapides comme celles que nous avons eues, notamment pour la euve $n^{0} 4$ (en 7 minutes), sont exagérées. Avec des laits acides eamme l'était le lait de cette cuve - mais dont l'acidité n'a pas été prise - nous aurions dû emprésurer au-dessous de $35^{\circ}$, à 30 ou $32^{\circ}$, pas plus, et mettre moins de présure, de façon à avoir une prise de caillé en 15 minutes au minimum, en 20 minutes au maximum.

Les quantités de présure que nous avons utilisées nous semblent bien trop importantes. On aurait pu les diminuer d'un tiers. Pour la première euve, la prise aurait eu lieu entre 20 et 25 minutes; pour la seconde, aux environs de 15 minutes; pour la troisième en $10 \mathrm{mi}$ nutes ; pour la quatrième en 7 minutes. On voit que pour la troisième et la quatrième cuves, la quantité de présure aurait même pu être ramenée au chiffre habituel, e'est-à-dire au tiers environ de ce qui a été employé.

En effet, pour la cuve $3, \mathrm{CO}^{2}+\mathrm{CaCl}^{2}$, où la prise a eu lieu en 7 minutes, avec la dose du tiers, la prise aurait eu lieu en 20 minutes ; pour la cuve 4 , où la prise a eu lieu en 5 minutes, elle aurait eu lieu, avec la dose du tiers, en 15 minutes. Dans ces conditions, les prises seraient évidemment normales. La présure se fixe sur le caillé, en grande partie, et joue un rôle dans la maturation, avec les ferments 
lactiques qui se trouvent à côté. L'excès de présure ne donne-t-il pas un goût qui n'est pas toujours très fin ?

L'impression que nous avons eue est done que nous avions affaire à des laits plutôt acides et que l'on employait trop de présure.

Il y a lieu de se féliciter de l'emploi du chlorure de calcium.

Nous préférerions beaucoup, pour que l'enseignement à tirer de ces résultats fut tout à fait complet, que l'on reprît toutes ces expériences, en notan't, avec beaucoup de précision, tout ce qui est fait. Nous voudrions que les fromages de chaque cuve fussent nettement séparés.

C'est à ce prix seul que l'on peut, évidemment, tirer des conclusions précises.

Avant que l'emploi du lait stassanisé ne soit commencé systématiquement pour la fabrication des fromages, il serait intéressant de travailler quatre jours de suite dans les conditions suivantes (nous disons quatre jours de suite pour que les conditions atmosphériques soient sensiblement les mêmes) :

Le premier jour, on travaillerait comme d'habitude, avec les quantités de présure, les températures, etc., auxquelles on avait recours jusqu'ici.

Le second jour, on travaillerait avec le lait qui aurait reçu un courant d'acide earbonique, sans doute pendant un temps plus long que 10 minutes, et en agissant un peu différemment.

Le troisième jour, on opérerait après addition de chlorure de calcium et passage du gaz carbonique.

Le quatrième jour, enfin, on opérerait avec du chlorure de calcium seul.

La comparaison serait alors parfaite, car nous pourrions, en effet, examiner le lait de toute une journée en face de celui des trois autres jours. De plus, cette comparaison ne devrait pas être globale. On devrait comparer les uns aux autres les fromages provenant des quatre premières cuves, puis, entre eux, ceux provenant des quatre secondes cuves, etc. Nous aurions de même à comparer sur l'ensemble des quatre jours, les fromages des premières à ceux des secondes, à ceux des troisièmes et à ceux des quatrièmes.

Nous n'avons pas à insister sur ce que peut avoir de fécond et d'instructif cette façon de procéder. Mais il reste bien entendu que, pour que nous en puissions tirer les meilleurs résultats, il est essentiel que toutes les indications que nous avons données plus haut soient suivies à la lettre. Tous les facteurs qui jouent dans l'emprésurage, dans le travail du caillé, dans le travail sous presse, etc., doivont être soigneusement relevés.

Si ces premiers essais sont loin de nous avoir autorisés à conclure 
ils nous ont permis de fixer le protocole des expériences qu'il convient de réaliser, en nous montrant les nombreux points auxquels il faut prêter attention. Pour bien comprendre ce qui se passe, éliminer les fausses mancuvres et espérer atteindre un heureux résultat que l'on peut dès maintenant entrevoir, il faut procéder, en fuyant les conclusions hâtives, avec toute la rigueur indispensable à la vraie recherche scientifique.

\title{
LA CORROSION DES MÉTAUX PAR LE LAIT
}

\author{
par \\ Colin G. FINK et Frederick A. ROHRMAN \\ de la Division d'Electrochimie de Columbia University (New-York).
}

(Fin.)

\section{DIFFERENCE DES DEGRÉS DE CORROSION}

Une étude détaillée de la corrosion dans une usine de ce type démontra que la corrosion du nickel était la plus prononcée dans les régénérateurs. Dans presque tous les eas, la corrosion la plus accentuée s'était produite dans cette partie de l'outillage : elle y était prononcée au point de creuser des anfractuosités et des crevasses profondes sur les parois des conduites. Dans les autres sections de l'usine, la corrosion des appareils de nickel n'était pas accentuée au même degré que dans les conduites des régénérateurs.

\section{UNE THEORIE DU DEGRÉ DE CORROSION}

Ainsi que nous l'avons déjà mentionné, en présence d'oxygène, il y a une tendance à la formation, à la surface du nickel, d'une couche protectrice d'oxyde, qui résiste à l'action corrosive. Récemment, BRown, Roetheli et ForRest [21] ont fait quelques déterminations intéressantes des degrés initiaux de corrosion de différents métaux, parmi lesquels le nickel et le cuivre. Leurs données démontrèrent, qu'en présence d'eau aérée, le nickel entrait beaucoup plus lentement en solution que le cuivre. En d'autres mots, la couche d'oxyde qui doit d'abord se former sur le nickel est moins soluble et protège mieux le métal qu'elle recouvre que la couche d'oxyde de cuivre. On ne peut oublier, qu'en présence d'oxygène, le potentiel du. métal est en réalité le potentiel de l'oxyde de ce métal. Dans ce cas, on ne peut faire un usage trop énergique de la série électromotive,

Comme ces effets de l'oxygène et des couches d'oxydes dans: l'eau sont sans doute approximativement les mêmes pour les métaux se trouvant dans le lait, nombre de eontradictions apparentes peuvent être expliquées. D'abord, le lait est froid et contient une grande quantité d'oxygène ; de ce fait, quand il passe par les appa- 